\title{
Matthias Pohlig, Marlboroughs Geheimnis
}

\section{Guido Braun}

\section{OpenEdition \\ Journals}

Édition électronique

URL : http://journals.openedition.org/ifha/8963

DOI : $10.4000 /$ ifha. 8963

ISSN : 2198-8943

Éditeur

IFRA - Institut franco-allemand (sciences historiques et sociales)

\section{Référence électronique}

Guido Braun, « Matthias Pohlig, Marlboroughs Geheimnis », Revue de l'IFHA [En ligne], Date de recension, mis en ligne le 29 novembre 2017, consulté le 24 septembre 2020. URL : http:// journals.openedition.org/ifha/8963; DOI : https://doi.org/10.4000/ifha.8963

Ce document a été généré automatiquement le 24 septembre 2020.

(C)IFHA 


\title{
Matthias Pohlig, Marlboroughs Geheimnis
}

\author{
Guido Braun
}

\section{RÉFÉRENCE}

Matthias Pohlig, Marlboroughs Geheimnis. Strukturen und Funktionen der Informationsgewinnung im Spanischen Erbfolgekrieg (Externa, vol. 10), Köln/Weimar/Wien: Böhlau, 2016, 457 p., $60 €$ 
Il ne fait aucun doute que l'histoire de l'espionnage connaît aujourd'hui un essor considérable. L'affaire autour de la NSA et d'Edward Snowden qui a éclaté en 2013 ainsi que l'évolution galopante des technologies de l'information confortent le sentiment d'une surveillance généralisée. Les hommes politiques et les spécialistes des technologies de l'information, mais aussi les juristes se trouvent confrontés au défi posé par l'espionnage et la surveillance. C'est ainsi qu'en 2016, dans une revue consacrée aux questions d'administration publique (Die öffentliche Verwaltung), un juriste allemand, Matthias Lachenmann, s'est intéressé à la surveillance numérique et s'est demandé si elle sonnait le glas de l'État de droit allemand. Si l'histoire ne

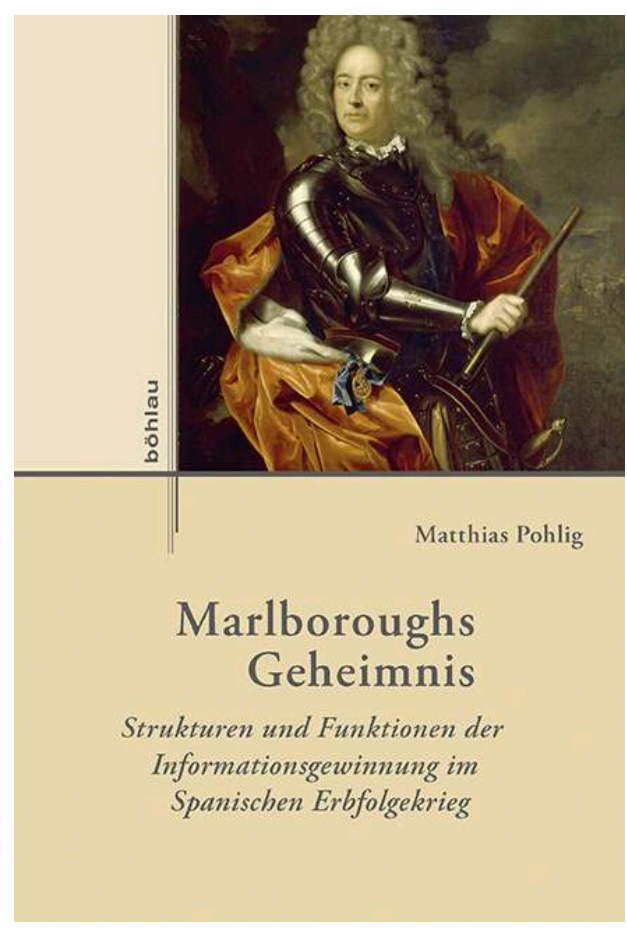
propose pas de solutions toutes prêtes à nos problèmes et à nos questions, elle peut néanmoins nous servir de source d'inspiration et nous aider à nous orienter. Ce sont en effet les défis sociaux actuels mais aussi des raisons immanentes à notre métier d'historien qui nous incitent aujourd'hui à réfléchir au rôle historique des espions, de l'espionnage et de la diplomatie secrète. Si nous nous penchons en particulier sur la période moderne, il est possible d'aborder ces problèmes en faisant abstraction de nos préoccupations actuelles.

Malgré une certaine intensification de la recherche ces dernières années, force est de constater que l'état de la recherche est encore insuffisant à de nombreux égards : les institutions et les centres de recherche ainsi que les revues et les bibliographies spécialisées qui se consacrent aux questions de l'espionnage privilégient les $\mathrm{XIX}^{\mathrm{e}}-\mathrm{XXI}^{\mathrm{e}}$ siècles, au détriment de la période moderne. De nombreuses questions fondamentales demeurent ouvertes. Il faut donc vivement saluer la publication, en 2016, de l'étude de Matthias Pohlig sur l'espionnage britannique au temps de la guerre de Succession d'Espagne. Il y a trente ans, Lucien Bély avait consacré sa thèse d'État à la diplomatie française à la fin du règne de Louis XIV, intitulée à juste titre «Espions et ambassadeurs ", tant les deux domaines du monde de l'espionnage et de la diplomatie s'interpénètrent. Le livre de $\mathrm{M}$. Pohlig vient compléter cette étude. Plus modeste du point de vue quantitatif (un peu plus de 450 pages), cet ouvrage n'en offre pas moins des résultats tout à fait considérables.

L'auteur a en effet mis à profit les fonds d'archives de la British Library, des Archives nationales de Kew et du British Postal Museum and Archive de Londres. La bibliographie des sources imprimées et des ouvrages et des articles consultés comprend plus de 70 pages. Ainsi l'auteur permet-il de mieux comprendre la quête d'information aux XVII ${ }^{\mathrm{e}}$ et $\mathrm{XVIII}^{\mathrm{e}}$ siècles. Par exemple, une question primordiale est de savoir qui agit comme espion à l'époque moderne. Quel est le rôle des diplomates "officiels ", des serviteurs, des soldats, des officiers, des migrants, des marchands, des ecclésiastiques, 
des femmes, etc.? Avons-nous affaire à un «métier d'espion» qui se constitue progressivement? Face à ces questions discutées par la recherche récente, M. Pohlig souligne que vers 1700 les réseaux d'information et d'informateurs se cristallisent autour de certains personnages influents, en particulier Marlborough, plutôt qu'autour de structures étatiques. De toute évidence, ce n'est qu'au cours du XVIII siècle que les premiers services institutionnels apparaissent en Europe, par exemple la chancellerie secrète à Vienne alors qu'ils n'existent pas encore au début du siècle.

De plus, M. Pohlig montre que l'espionnage fait partie d'un système plus vaste visant à obtenir des informations. L'auteur se montre peut-être un peu trop réticent à utiliser les termes d'« espion » et d'« espionnage " qui, pourtant, sont très utiles malgré les différences par rapport aux services de renseignements institutionnalisés et aux espions professionnels que nous connaissons de nos jours. Or, même si les diplomates et d'autres informateurs collectent des informations diverses, une partie de leurs tâches relève très certainement de l'espionnage dès lors que les arcanes du pouvoir s'intéressent à leurs activités.

Le premier chapitre du livre (45 pages) est consacré aux conditions qui encadrent l'action diplomatique au début du XVIII ${ }^{\mathrm{e}}$ siècle (causes, déroulement et résultats de la guerre de Succession d'Espagne, biographie de Marlborough). Dans le deuxième chapitre, le plus long du livre (215 pages), l'auteur analyse les structures de la quête d'information, son organisation, le rôle des réseaux d'informateurs, de la poste et de la presse, etc. Le troisième chapitre, quoique plus court (70 pages), est peut-être la partie la plus intéressante de l'ouvrage car l'auteur y expose les différentes fonctions de l'information. Il s'avère que l'information est certes utile pour l'élaboration de décisions politiques ou militaires, mais c'est loin d'être la seule fonction de l'information au début du XVIII ${ }^{e}$ siècle.

En effet, la collecte d'informations, et surtout d'informations exclusives, fait partie des stratégies de représentation et d'autoreprésentation de personnages politiques tels que Marlborough, la connaissance du secret les assimilant à l'image du prince détenteur des arcanes de l'État. Être bien informé, c'est se montrer compétent. La compétence avérée sert à légitimer le rang social et politique. En même temps, les informations constituent une sorte de monnaie susceptible d'être échangée contre d'autres renseignements ou des biens (au sens propre comme au sens figuré). Leur échange contribue ainsi à l'établissement ou à la consolidation de systèmes de clientèles. Ces fonctions multiples, au-delà de la prévention et de la prise de décisions, expliquent la « faim d'information » qui caractérise (entre autres) les contemporains au début du XVIII siècle, pour reprendre l'expression de Lucien Bély.

M. Pohlig a le mérite d'avoir dégagé les structures de la production d'informations et de leurs fonctions (politiques, militaires, sociales, culturelles) en Angleterre vers 1700. Souhaitons que ce livre contribue à une intensification de la recherche dans ce domaine. En effet, de nombreuses questions restent en suspens. Malgré l'étude fondamentale de Lucien Bély et les précieuses recherches d'autres historiens français comme Alain Hugon et Serge Brunet, par exemple, nous sommes encore loin de connaître dans le détail le fonctionnement de l'espionnage et de la diplomatie secrète à l'époque moderne: la formation des réseaux d'informateurs, les techniques de sécurisation des informations, les normes qui ont régi le comportement et la communication des différents acteurs concernés, le recrutement des espions et les aspects transculturels de l'espionnage, les problèmes que l'espionnage pose au niveau 
de la communication linguistique, symbolique et interculturelle, etc. sont autant de sujets à approfondir.

INDEX

Index chronologique : Période moderne

Thèmes : Histoire des États et des pouvoirs

\section{AUTEURS}

\section{GUIDO BRAUN}

Rheinische Friedrich-Wilhelms-Universität Bonn 